\title{
The DAMPE experiment: performances and results
}

\author{
Antonio De Benedittis*t \\ Dip.to di Matematica e Fisica "E. De Giorgi", Università del Salento, and Istituto Nazionale di \\ Fisica Nucleare (INFN), Sezione di Lecce, Via per Arnesano, 73100, Lecce, Italy \\ E-mail: antonio.debenedittisale.infn.it
}

The DAMPE (DArk Matter Particle Explorer) experiment is a space mission whose main purpose is the detection of cosmic electrons and photons up to energies of $10 \mathrm{TeV}$, in order to identify possible evidence of Dark Matter in their spectra. Furthermore it aims to measure the fluxes and the elemental composition of the galactic cosmic rays nuclei up to $100 \mathrm{TeV}$ and more, in order to get a better understanding of the galactic sources, acceleration mechanisms and propagation processes in the Galaxy. DAMPE is supported by an international collaboration involving universities and scientific institutions of the People's Republic of China, of Italy and the University of Geneva. The satellite was successfully launched on December 17th, 2015 from the Jiuquan Satellite Launch Center. The DAMPE detector consists of: a double layer of Plastic Scintillator Detector; a Silicon-tungsten Tracker-converter; an electromagnetic calorimeter (composed by BGO crystals) and a Neutron Detector.

This is the first experiment which provides a direct measure of the electron spectrum up to energy higher than $10 \mathrm{TeV}$ and of the cosmic ray nuclei spectra up to $\sim 100 \mathrm{TeV} / n$ with high statistics. The electron plus positron measured spectrum confirms the cut-off at $\sim 1 \mathrm{TeV}$ according to the HESS measurement, whereas the proton and helium spectra show the spectral hardening at hundreds of $\mathrm{GeV}$ in agreement with the past measurements carried out by previous experiments; moreover in the proton spectrum it was found a softening at $\sim 14 \mathrm{TeV}$. These results suggest the existence of spectral features of cosmic rays at energy below the so-called knee.

As concern the gamma astronomy, DAMPE has reconstructed a very precise sky map identifying a very high number of pulsars.

We intend to present and discuss the performances of the detector and the main scientific results obtained after three years of data taking.

European Physical Society Conference on High Energy Physics - EPS-HEP2019 -

10-17 July, 2019

Ghent, Belgium

\footnotetext{
* Speaker.

${ }^{\dagger}$ On behalf of the DAMPE collaboration
} 


\section{Introduction}

Cosmic Rays (CRs) are widely to be believed originated by the strong shock of Supernovae explosions in the Milky Way [1]. The CRs energy spectrum is expected to follow a featureless power-law, in a range between few tens of $\mathrm{GeV}$ up to about $3 \mathrm{PeV}$ (the so-called knee-region [2]) according to the conventional acceleration models [3]. Recent measurements of proton and helium nuclei fluxes carried out by baloon- and space-borne experiments (ATIC-2 [4], PAMELA [5], AMS-02 [6, 7], CREAM I-III [8], NUCLEON [9] and CALET [10]), observed unexpected spectral features, that is deviations from the single power-law distribution. In particular it was observed a spectral hardening at energies of about $200 \mathrm{GeV} / n$, and a spectral softening at energies higher than $50 \mathrm{TeV} / n$.

High-energy Cosmic Ray electrons and positrons (CREs) flux also differs from the simple power law showing structures at energies of the order of $\mathrm{TeV}$, in fact as observed by HESS [11, 12] at about $1 \mathrm{TeV}$ the energy spectrum present a cut-off followed by a new increase.

Moreover the detector is also suitable for the study of the high-energy behavior of gamma-ray bursts, pulsars, Active Galaxy Nuclei and other transients.

A precise measurement of all these features is fundamental for the study of phenomena related to the acceleration and the propagation of galactic CRs, and to highlight the presence of nearby sources or dark matter. The possibility of making these measurements is guaranteed by the onorbit calibration that highlights the good operation and stability of each sub-detector [13].

\section{DAMPE Detector}

The DAMPE [14] detector is composed from the top to the bottom by:

- a Plastic Scintillator strip Detector (PSD) whose goal is the measurement of the absolute value of the electric charge of incident particles (up to $Z \leq 28$ ), moreover it acts as an anticoincidence system for photons;

- a Silicon-Tungsten tracKer-converter (STK) that reconstructs the trajectory of incident particles, provides a redundant measurement of the charge and acts as a photon converter;

- a $B G O$ calorimeter whose depth (about 32 radiation lengths $X_{0}$ or equivantely 1.6 interaction lengths $\lambda_{I}$ ) ensures a high resolution measurement of the energy deposited by electrons, photons and nuclei, also providing an image of the shower useful to provides a good electron/hadron discrimination;

- a NeUtron Detector (NUD) that provides further information about the nature of the shower (electromagnetic or hadronic) using the neutrons produced by the hadronic showers.

\section{CRE Spectrum}

The measurement of the electron plus positron energy spectrum is made difficult by the abundant background formed by the proton flux. In order to overcome this issue and to identify correcly CREs, DAMPE experiment adopts a method based on a pattern recognition, namely it uses the 
shower profiles in the BGO calorimeter to discriminate electrons from protons. This method consists in the evaluation of the shower spread which is described as an energy dependent root mean square of hit positions in the calorimeter:

$$
R M S_{i}=\sqrt{\frac{\sum_{j}\left(x_{j, i}-x_{c, i}\right)^{2} \cdot E_{j, i}}{\sum_{j} E_{j, i}}}
$$

in which $x_{j, i}$ and $E_{j, i}$ are the coordinates and the deposited energy of the $j$-th bar in the $i$-th layer respectively, and $x_{c, i}$ is the coordinate of the shower centre of the $i$-th layer. In Figure 1 (a) the ratio $\mathscr{F}_{\text {last }}$ between the energy deposited in the last layer of BGO over the total deposited energy, used to describe the tail of a shower, is plotted as a function of the total root mean square $\left(\sum_{i} R M S_{i}\right)$. This method together with the event pre-selection procedures ensures the rejection of about $99.99 \%$ of proton candidates.

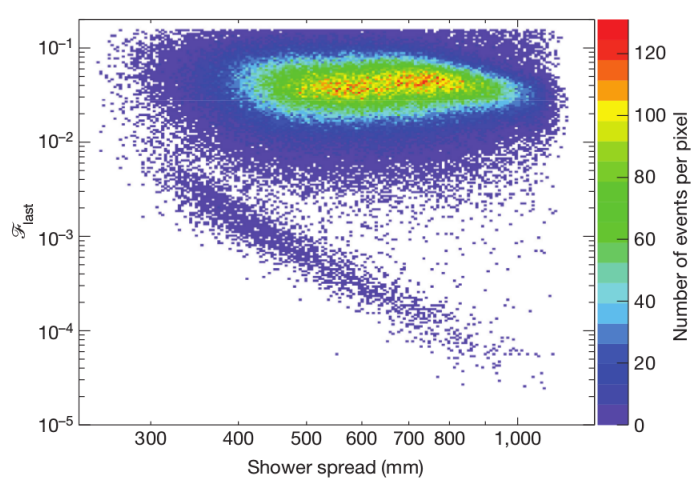

(a)

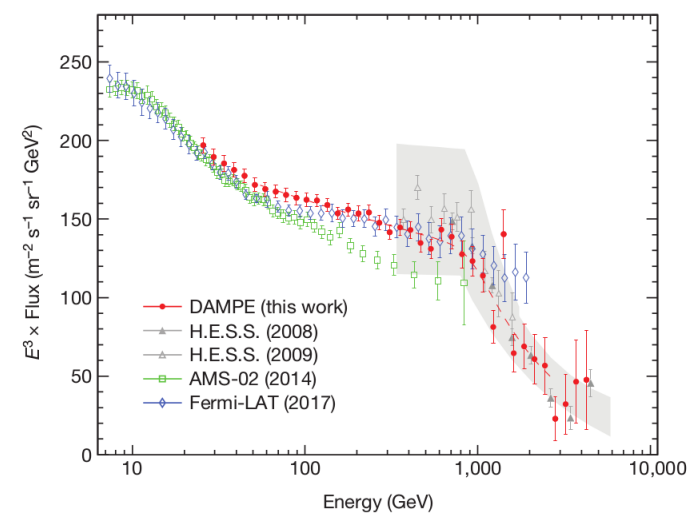

(b)

Figure 1: (a) Last layer energy fraction $\mathscr{F}_{\text {last }}$ as a function of the shower spread $\sum_{i} R M S_{i}$ for event selected with deposited energy between $500 \mathrm{GeV}$ and $1 \mathrm{TeV}$. (b) The CRE spectrum measured by DAMPE [15] compared with the previous measurements of AMS-02 [16], HESS [11, 12] and FERMI-LAT [17]. The gray band represent the systematic errors of HESS measurement.

With the data collected in 530 days of flight, we obtain a very precise measurement of the CRE spectrum from $25 \mathrm{GeV}$ to $4.6 \mathrm{TeV}$ (Figure 1 (b)). The DAMPE result reveals a spectral break at energies of about $0.9 \mathrm{TeV}$, with a variation in the spectral index from -3.1 to -3.9 , according to the indirect measurement carried out by HESS, which was affected by relatively large systematics.

\section{Gamma-ray astronomy}

Gamma-ray astronomy is another of the scientific objectives of the experiment. Thanks to its high energy resolution, DAMPE plays a key role in the observations of $\gamma$-ray transients, revealing the nature of these phenomena which are very important in the multi-messenger era, providing more information in particular in conjunction with observation of gravitational waves and very high energy astrophysical neutrinos.

During three years of data-taking a high resolution sky map was obtained (Figure 2), and a sample of more than 20 pulsars was identified. 

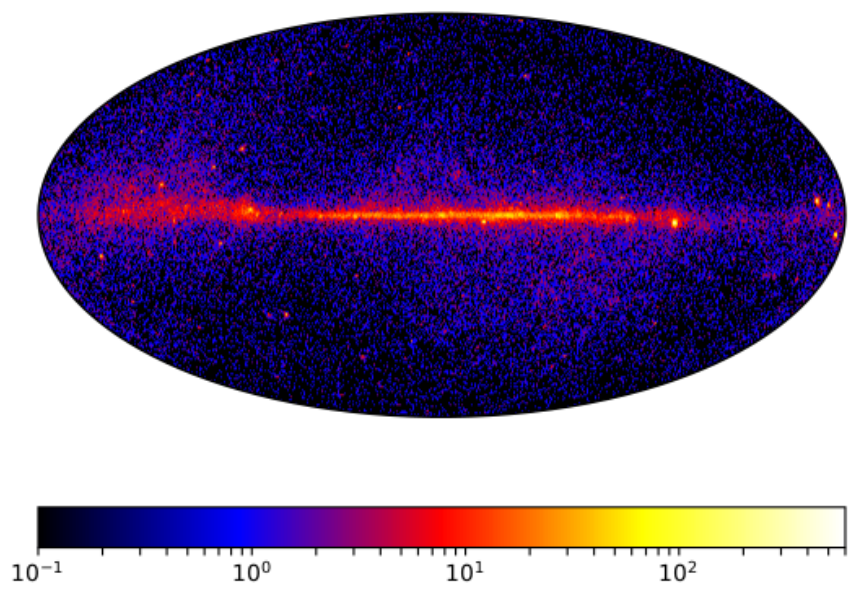

Figure 2: $\gamma$-ray sky observed by DAMPE in three years of data-taking in the energy range between $1 \mathrm{GeV}$ and $1 \mathrm{TeV}$.

\section{Proton and Helium nuclei spectra}

DAMPE has a high capability in the charge selection (the charge resolution in terms of Gaussian standard deviation is 0.06 for protons and 0.30 for iron nuclei), this allows to measure the energy spectra of CRs with very high precision.

For the analysis of the proton CRs component a dataset of 30 months was used (from January 1, 2016 to June 30, 2018). The live time is about the $75.5 \%$ of the total exposure time after subtracting the instrumental dead time, the crossing time of the South Atlantic Anomaly region, the time for the calibration procedures. The selected events have to satisfy the following requests: almost $20 \mathrm{GeV}$ of deposited energy in BGO, geometric containment of the track in all the subdetectors, high energy trigger condition. Finally the proton candidates sample is selected applying a cut on the charge measured by the PSD as a function of the deposited energy in BGO. In Figure 3 the PSD charge distribution of low $Z$ nuclei events between 447 and $562 \mathrm{GeV}$ compared with the $\mathrm{MC}$ for protons and Helium nuclei is shown. The vertical line shows the cut applied to select proton candidates. MC samples are useful to estimate the Helium background, which is less than $1 \%$, and also to estimate the selection efficiencies of each analysis cut, moreover they are important as they allow to obtain the response matrix of proton events in the calorimeter and the effective acceptance defined as:

$$
A_{e f f, i}=A_{g e n} \times \frac{N_{\text {pass }, i}}{N_{\text {gen }, i}}
$$

in which $A_{g e n}$ is the geometrical acceptance of the MC generation half-sphere, $N_{p a s s, i}$ and $N_{g e n, i}$ are the number of generated and surviving events, respectively.

In Figure 4 the effective acceptance and the proton spectrum from $40 \mathrm{GeV}$ to $100 \mathrm{TeV}$ are shown. The result confirms the presence of the hardening at $E \simeq 200 \mathrm{GeV}$ in agreement with the measurements of AMS-02 and PAMELA. Moreover a softening change in the spectral index from -2.60 to -2.85 at $E \simeq 14 \mathrm{TeV}$ was observed with unprecedent resolution. 


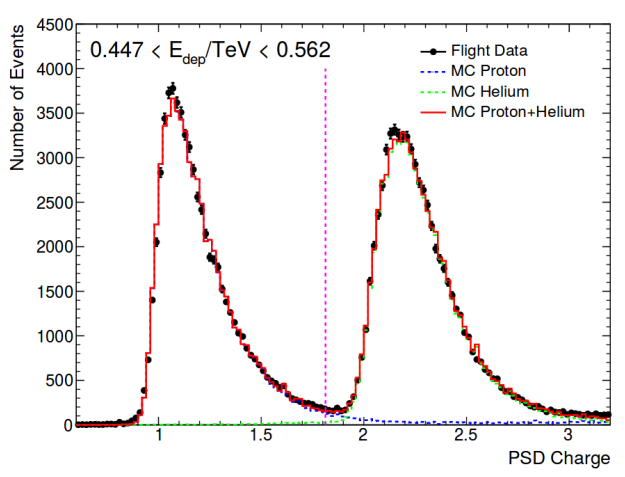

Figure 3: The combined PSD charge spectrum for protons and helium nuclei, for BGO energies between $447 \mathrm{GeV}$ and $562 \mathrm{GeV}$. In this plot on-orbit data (black) are compared with the MC protons (blue) and MC helium nuclei (green).

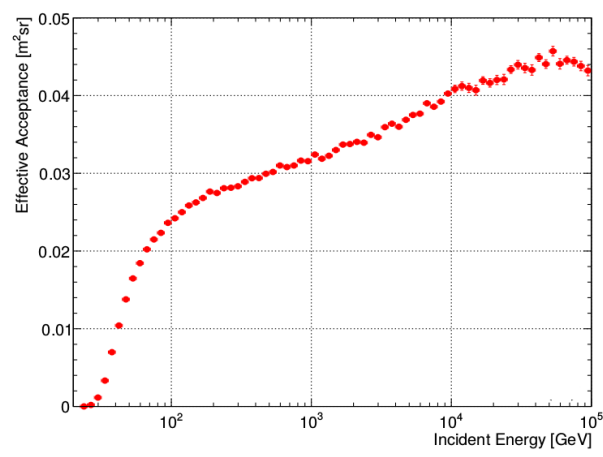

(a)

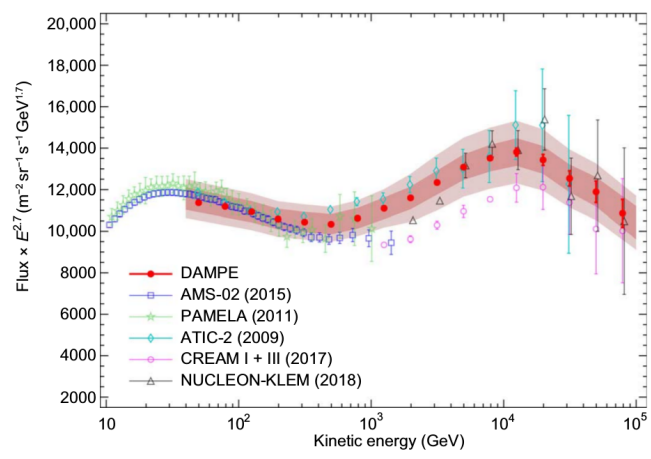

(b)

Figure 4: (a) Effective acceptance of protons as a function of primary energy. (b) Proton energy spectrum measured by DAMPE [18], compared with the previous measurement by AMS-02 [6], PAMELA [5],CALET [10], ATIC [4], CREAM I-III [8], NUCLEON [9].

A similar analysis was carried out for the Helium nuclei flux. In Figure 5 the Helium effective acceptance and flux in the range between $10 \mathrm{GeV} / n$ and $5 \mathrm{TeV} / n \mathscr{F}$ last are shown. Also in this case a spectral hardening is clearly visible at hundreds of $\mathrm{GeV} / n$ which is consistent with the previous measurements.

\section{Summary}

The DAMPE mission is working in a stable data taking since few day after December 17, 2015. Since then it has collected over 6 billion CR events. Precise measurement of the CRE spectrum up to energies of $4.6 \mathrm{TeV}$ has been obtained, observing a break at $0.9 \mathrm{TeV}$ with higher precision compared with previous measurements. The CR proton flux from $40 \mathrm{GeV}$ to $100 \mathrm{TeV}$ has been measured, confirming the spectral hardening at hundreds of $\mathrm{GeV}$ and revealing a spectral softening at $13.6 \mathrm{TeV}$ leading to interesting implications in the acceleration and propagation mechanisms of CRs. A preliminary Helium flux has been measured from $10 \mathrm{GeV} / n$ to $5 \mathrm{TeV} / n$ observing also in this case a spectral hardening at hundreds of $\mathrm{GeV} / n$. The data analysis is still on-going and in the 


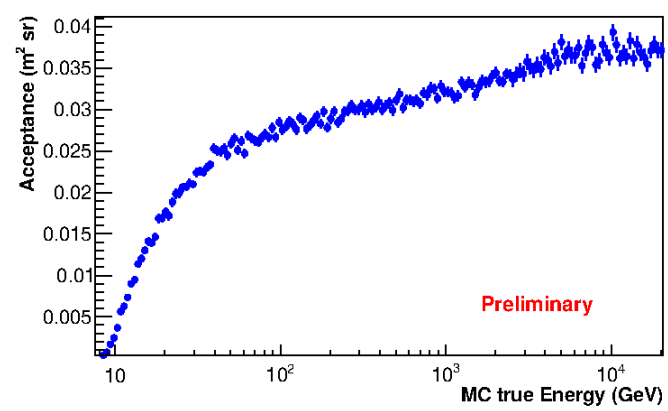

(a)

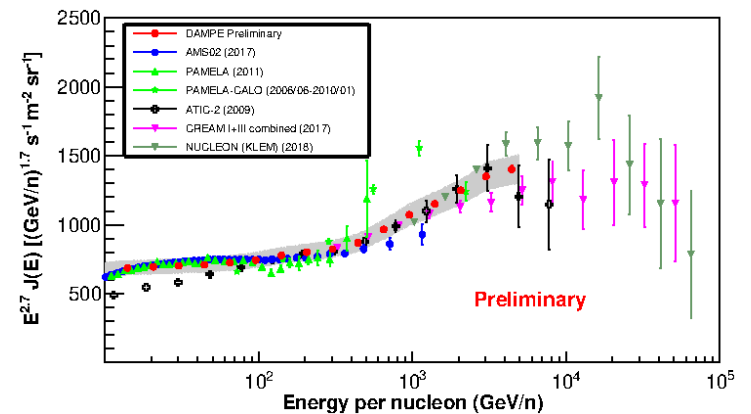

(b)

Figure 5: (a) Effective acceptance of Helium as a function of primary energy. (b) Helium energy spectrum measured by DAMPE [19], compared with the previous measurement of AMS-02 [7], PAMELA [5], ATIC [4], CREAM I-III [8], NUCLEON [9].

future, with the increase in data sample it is expected to obtain fluxes up to energies even higher than those achieved, providing important information in order to increase our knowledge of very high energy astrophysical phenomena.

\section{References}

[1] Baade W., Zwicky F., Phys. Rev. 46, 76 (1934).

[2] Amenomori M. et al. (The Tibet AS $\gamma$ Collaboration), Astrophys. J. 678, 1165, (2008).

[3] Fermi E., Phys. Rev., 75, 1169 (1949).

[4] Panov A. D. et al. (The ATIC Collaboration), Bull. Rus. Acad. Sci. Phys. 73, 564 (2009).

[5] Adriani O. et al. (The PAMELA Collaboration), Science 332, 69 (2011).

[6] Aguilar M. et al. (The AMS Collaboration), Phys. Rev. Lett. 114, 171103 (2015).

[7] Aguilar M. et al. (The AMS Collaboration), ,Phys. Rev. Lett. 115, 211101, (2015).

[8] Yoon, Y. S. et al. (The CREAM Collaboration), Astrophysical J. 839, 5, (2017).

[9] Atkin E. et al. (The NUCLEON Collaboration), Astropart. Phys. 90, 69-74 (2017).

[10] Adriani O. et al. (The CALET Collaboration), Phys. Rev. Lett. 122, 181102, (2019).

[11] Aharonian F. et al. (The H.E.S.S. Collaboration), Phys. Rev. Lett. 101, 261104, (2008).

[12] Aharonian F. et al. (The H.E.S.S. Collaboration), Astron. Astrophys. 508, 561, (2009).

[13] Ambrosi G. et al. (The DAMPE Collaboration), Astropart. Phys. 106, 18, (2019).

[14] Chang J. et al. (The DAMPE Collaboration), Astropart. Phys. 95, 6-24 (2017).

[15] Ambrosi G. et al. (The DAMPE Collaboration), Nature 552, 63, (2017).

[16] Aguilar M. et al. (The AMS Collaboration), Phys. Rev. Lett. 113, 221102, (2014).

[17] Abdollahi S. et al. (The Fermi-LAT Collaboration), Phys. Rev. D95, 082007, (2017).

[18] An Q. et al. (The DAMPE Collaboration), Sci. Adv. 5, 9, (2019).

[19] Di Santo M. et al., in proceedings of 36th International Cosmic Ray Conference, POS (ICRC2019) 058, (2019). 